\section{Sprachstörung bei Demenz}

\section{Eine Sprachstörung bei Demenz hat ein oft-} mals heterogenes Erscheinungsbild. Allerdings unterscheiden sich die sprachlichen Symptome je nach Demenztyp.

Das klinische Syndrom der Demenz wird als Oberbegriff gesehen für verschiedene Demenztypen, wobei je nach Demenztyp unterschiedliche Gehirnareale bevorzugt von einer Atrophie befallen sind. Dementsprechend werden auch unterschiedliche kognitive Symptome deutlich, die schleichend beginnen, langsam fortschreiten und eine deutliche Beeinträchtigung im Alltag nach sich ziehen. Die AlzheimerDemenz (AD) stellt den am häufigsten auftretenden Demenztyp dar. Sprach- bzw. Kommunikationsstörungen sind ein typisches Symptom der AD, wobei jedoch auch weitere nonverbale kognitive Fähigkeiten betroffen sind, wie z. B. Gedächtnis, Aufmerksamkeit, Orientierung, logischen Denken und Urteilen, sowie planvollem Handeln. Ebenso beobachtet man Veränderungen des Antriebs und der Affektkontrolle.

Weniger bekannt ist der Demenztyp der primär progressiven Aphasie (PPA). Eine PPA zeichnet sich aus durch eine langsam fortschreitende Störung der Sprache bei relativem Erhalt nonverbaler kognitiver Fähigkeiten.

\section{Sprachliche Symptomatik \\ $\nabla$}

Eine Sprachstörung bei AD zeigt sich relativ heterogen, trotzdem kann ein gewisser Verlauf der Symptome erkennbar sein. Oft früh beeinträchtigt sind pragmatische Fähigkeiten, z.B. eine fehlende Anpassung an den Gesprächspartner. Auch treten bereits Wortfindungsstörungen auf und das syntaktische Verständnis kann beeinträchtigt sein. Im Verlauf der Erkrankung wird die Sprache zunehmend inhaltsleerer, es können sich u.a. semantische Paraphasien und Störungen im Verständnis einfacher Sätze bzw. Einzelwörter zeigen. Im fortgeschrittenen Stadium der Erkrankung zeigen sich üblicherweise massive Beeinträchtigungen sowohl in der Sprachproduktion als auch im Sprachverständnis.
Die PPA vereint 3 symptomatisch unterschiedliche Varianten unter sich:

- Die agrammatische bzw. nichtflüssige Variante (avPPA) zeigt eine Störung der Grammatik und / oder eine unflüssige Spontansprache mit Sprachanstrengung (diagnostisch zwingend notwendig muss nur eines der Symptome auftreten).

- Die semantische Variante ist gekennzeichnet durch den Verlust der semantischen Fähigkeiten, die sich v.a. im Benennen und Verstehen von Einzelwörtern und dem Objekterkennen auswirken.

- Die logopenische Variante zeigt neben Wortfindungsstörungen auch Störungen des Nachsprechens von Sätzen/Phrasen.

\section{Diagnostik}

$\nabla$

Die Diagnose des genauen Demenztyps ist wichtig, um Kenntnis über den weiteren symptomatischen Verlauf und mögliche Ressourcen des Patienten zu erhalten. Dieses Wissen ist grundlegend für die Festlegung der Therapieinhalte. Beispielsweise ist es wichtig, ob neben der Sprachstörung auch weitere kognitive Defizite vorliegen, die es in der Therapie zu berücksichtigen gilt. In der Sprachdiagnostik wird in Ermangelung demenzspezifischer Tests auf „klassische“ Aphasietests zurückgegriffen, die oftmals unzureichend für das Störungsbild der dementiell bedingten Sprachstörung validiert sind.

\section{Therapie \\ $\nabla$}

Ziel der Therapie von Sprachstörungen bei Demenz ist weniger eine Verbesserung der Symptomatik als der Erhalt des Status quo bzw. einer Verzögerung des Sprachabbaus. Eine Therapie sollte nicht störungsspezifisch sondern ressourcenorientiert erfolgen, um noch bestehende Fähigkeiten zu festigen.
Ebenfalls ein wichtiges Ziel stellt die Aufrechterhaltung der Kommunikation im Alltag durch kommunikative Strategien und Hilfestellungen dar. Hierbei ist die Aufklärung und Anleitung der Angehörigen zentral.

\section{Audiobeispiel \\ $\nabla$}

Die Audioaufnahme ${ }^{1}$ des 70-jährigen, männlichen Patienten mit avPPA entstand ca. 2 Jahre nach dem ersten Auftreten sprachlicher Symptome. Eine Kommunikation gelingt zu diesem Zeitpunkt bereits nur mit Unterstützung des Gesprächspartners. Es zeigt sich für die avPPA typische Sprachanstrengung und ein stockender Redefluss. Ein Agrammatismus ist zu diesem Zeitpunkt nicht vordergründig beobachtbar. Eine sprechapraktisch anmutende Artikulationsproblematik kann ebenfalls begleitendes Symptom bei avPPA sein.

\section{Fazit}

Sprachstörungen bei Demenz sind sowohl symptomatisch als auch bezüglich der therapeutischen Herangehensweise von „klassischen“ vaskulär bedingten Aphasien abzugrenzen.

Dr. Christina Knels, Hamburg

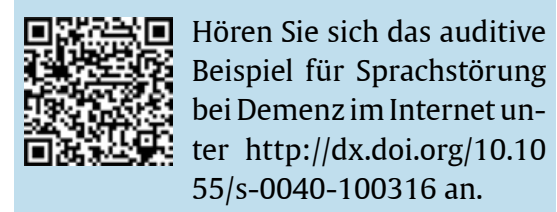

${ }^{1}$ Aufnahme: Thomas Brauer, Lehrlogopäde, Universitätsmedizin Mainz, mit freundlicher Genehmigung. 